\title{
RESPONSE
}

\section{Infant perseveration: Rethinking data, theory, and the role of modelling}

\section{Yuko Munakata}

University of Denver, USA

The commentaries on my model of the $\mathrm{A} \overline{\mathrm{B}}$ task highlight disagreements in the field at three levels: 1) data, 2) theory, and 3) the role of modelling. Some of the data disagreements have actually been resolved by existing studies. I will not venture to claim that any of the debates about theory and the role of modelling have been resolved, but I will discuss additional data and arguments and attempt to clarify my stance on these issues.

\section{Data}

The commentaries highlight five disagreements about data on the $A \bar{B}$ error: 1) Do looking measures show greater sensitivity than reaching measures? 2) Do infants perseverate to A when an object is visible at $B$ just as they would if the object were hidden? 3) Does increasing the number of hiding locations help infant performance for non-artifactual reasons? 4) Does increasing the number of $\mathrm{A}$ trials increase the likelihood of the $A \bar{B}$ error? and 5) Do infants show a U-shaped pattern of development in their $A \bar{B}$ errors? I will argue that existing data answer Question 1 in the affirmative and Question 2 in the negative, whereas additional work may be needed to resolve the remaining questions.

\section{Looking vs. Reaching}

Diamond challenges some of the studies I cited as evidence for earlier sensitivity in violation-of-expectation variants of the $A \bar{B}$ task (Baillargeon \& Graber, 1988; Baillargeon, DeVos, \& Graber, 1989), arguing that these were not equivalent to reaching versions of the task. Smith \& Scheier go one step further to challenge the general claim that looking measures show greater sensitivity than reaching measures, arguing that looking and reaching are strongly coupled and the greater sensitivity claim is primarily based on 'anecdotal reports that babies sometimes look correctly but reach wrong and on experimental evidence from nonreaching tasks.' These are important cautions, but the collection of data on looking and reaching measures of $A \bar{B}$ demonstrate that these behaviors, while strongly coupled, nonetheless show differential sensitivity in equivalent versions of the $\mathrm{A} \overline{\mathrm{B}}$ task. This point is made perhaps most clearly in Ahmed and Ruffman's (1997) expectation-reach studies and Hofstadter and Reznick's (1996) gaze-reach study. In Ahmed and Ruffman's (1997) studies, infants were presented with equivalent expectation and reach variants of the $\mathrm{A} \overline{\mathrm{B}}$ task; they were presented with an $A$ trial and allowed to reach and then presented with a $B$ trial, and either allowed to reach (generally reaching perseveratively) or watch as the toy was revealed at $A$ or $B$ (generally looking longer when the toy was revealed at A). In Hofstadter and Reznick's (1996) study, infants were presented with a standard $A \bar{B}$ task in which they were allowed to reach. Their direction of reach and gaze were the same on most trials, but when they differed, infants were more likely to gaze correctly while reaching incorrectly than vice versa. Thus, the claim that infants show greater sensitivity in looking measures of $A \bar{B}$ than in reaching is not based simply on non-equivalent task variants, anecdotal data, or non-reaching tasks. Finally, to clarify the network's performance in this regard, it does not fail 'to explain the close coupling of looking and reaching' as Smith \& Scheier argue. Instead, as Figure 6 in the original paper shows, the network's looking and reaching, like infants', are closely coupled (because they share common patterns of input activity) and yet show differential sensitivity (due to the gradedness of underlying representations and the different frequencies of updating in the two systems). 


\section{Errors with Visible Objects}

Bremner and Diamond mention the finding that infants occasionally search perseveratively at $A$ even when the object is visible at $B$ (Butterworth, 1977; Harris, 1974; Bremner \& Knowles, 1984), and use it (albeit in rather different ways) to challenge various theories of the $A \bar{B}$ error. However, as I discussed in the original paper, such rare errors reflect random incorrect responses rather than true, perseverative $A \bar{B}$ errors. Sophian and Yengo (1985) demonstrated this in a study including a third, control location. When the toy was visible at $B$ and infants erred, they were as likely to search at the control location as at the $A$ location. In contrast, when the toy was hidden at $B$ and infants erred, they were more likely to search perseveratively at the $A$ location than at the control location. Thus, an inability to account for the rare, random incorrect responding of visible-toy errors is unlikely to expose fundamental flaws in theories of the qualitatively different, perseverative responding of the $\mathrm{A} \overline{\mathrm{B}}$ error.

\section{Number of Hiding Locations}

Diamond and Reznick argue that infants show improved performance with multiple wells for an artifactual reason (only the correct well is uncovered and recovered in some multiple-well studies, whereas both wells are covered simultaneously in some two-well studies). Further, Diamond elaborates on her relevant data showing that performance on multiple-well studies is worse when all wells are covered simultaneously than when only the correct well is covered (Diamond, Cruttenden, \& Neiderman, 1994). As I argued in the original paper, this procedural difference may thus contribute to infants' improvements with multiple hiding locations. However, demonstrating that one artifactual factor influences infant performance does not mean that the entire effect is artifactual. In the very least, one would want to test whether controlling for the artifact leads to identical performance on equivalent multiple- and two-well studies. When this has been done (Bjork \& Cummings, 1984), the multiple-well advantage remains, so other factors must contribute to infants' improved performance with multiple wells. My model incorporates two such possible factors: additional locations reduce the salience of $A$, and additional locations favor non-prepotent responses.

\section{Number of A trials}

Diamond argues that the number of $A$ trials does not affect the likelihood of the $A \bar{B}$ error, and that this is a problem for both her theory and mine. However, as I argued in the original paper, the data are mixed on this issue; Wellman, Cross, and Bartsch's (1986) meta-analysis did not show any effects of number of $A$ trials, but subsequent studies have demonstrated clear effects of the number of reaches to A (Smith, McLin, Titzer, \& Thelen, 1995; Diedrich, Thelen, Smith, \& Corbetta, 1997). In addition, Bremner points out that equivocal data on the effects of distinct covers can be understood in terms of the effects of the number of $A$ trials, and that his own work with distinct covers demonstrates effects of the number of $A$ trials. Thus, further work is needed to clarify why and when effects of number of A trials are observed.

\section{U-shaped Pattern of Development}

Bremner provides anecdotal confirmation of my model's prediction that infants will show a U-shaped pattern of development with the $A \bar{B}$ error (showing increases in perseveration before showing decreases). This was heartening to read, but it is not yet clear how to resolve this confirmation with Diamond's disconfirmation from her systematic, longitudinal study of infants from an early age, at frequent intervals. One possibility is that Diamond's systematic manipulation of delay intervals (both between and within testing sessions) based on infant performance, while clearly and elegantly demonstrating developmental changes in tolerable delays, may have nonetheless obscured increases in perseveration that might have been evident if constant delays had been used across age groups.

\section{Theory}

The commentaries highlight four disagreements about theorizing relevant to the $A \bar{B}$ error: 1) What is the relationship between existing memory distinctions and the active-latent distinction I have proposed? 2) What is the relationship between our theorizing about infants vs. adults? 3) What does the $A \bar{B}$ task measure?, and 4) What is the role of reinforcement in infants' performance on the $A \bar{B}$ task? I believe that the first three questions are still open to debate, but available data suggest that reinforcement does not play a major role in infants' $A \bar{B}$ performance.

\section{Existing memory distinctions}

Mandler argues that my active-latent distinction 'ignores the last 50 years of research on memory in psychology and neuroscience' and that my 'wholesale revision of the field is quite alarming'. I am grateful to Mandler for 
pointing out this apparent problem, so that I can clarify what I meant when I wrote that active representations are related to notions of recall, working and short term memory, and latent representations are related to notions of recognition, habit and long term memory. Rather than suggesting a wholesale revision of the field in which these kinds of memory are lumped together (such a revision would in fact ignore the last 50 years of relevant research), I meant to argue that active and latent representations may be differentially (but not exclusively) tapped by distinct memory processes that have been elucidated in existing dichotomies. For example, the process of recall may require the calling to mind of a memory in the form of an active representation, whereas some recognition processes could rely on facilitated processing based on latent representations. Similarly, the temporary maintenance and/or manipulation of information in short term or working memory may require active representations that can be accessed by other systems, whereas habit and long term memories may differentially rely more on latent representations than on the maintained firing of neurons. Of course, as Mandler argues, these existing dichotomies do not map directly onto one another (e.g., both recall and recognition are processes that operate on long term memory representations). Similarly, I do not mean to argue that my proposed distinction between active and latent representations maps directly onto existing dichotomies. For example, although long term memories may rely on latent representations more than short term memories do, such long term latent representations can be used in the service of forming active representations during recall from long term memory. Similarly, although working memories may rely on active representations more than habit memories do, the ability to maintain such active working memory representations relies upon the appropriate configuration and strength of latent representations. ${ }^{1}$ Such interactions between active and latent traces may be largely responsible for the difficulty of cleanly relating the active-latent distinction to existing processing and memory dichotomies. However, the primary goal of this work is not to implement such existing dichotomies, but to understand how underlying mechanisms (active and latent) can give rise to perseverative behavior.

\section{Infants vs. adults}

Mandler argues that a good first hypothesis for why infants perseverate is the same reason that adults do, and

\footnotetext{
${ }^{1}$ These examples of how latent traces influence active traces should answer Diamond's question about whether active and latent representations interact: They do.
}

she provides the example of adults continuing to look for their watch on the usual wrist when wearing it on the other (for discussion of such everyday action errors, see Norman \& Shallice, 1986; Roberts \& Pennington, 1996). My interest is in what allows these adults to eventually learn to look to the correct wrist. Are they developing better inhibitory abilities? Or are they developing a stronger representation of the watch in its actual location? My argument is that the former may be a consequence of the latter, in both adults and infants (Roberts, Hager, \& Heron, 1994); the general principles of active-latent dynamics and graded representations embodied in the model would allow for its extension to account for adult errors. The further question of what infants and adults 'know' is debatable. To illustrate, Mandler argues that it is parsimonious to assume that infants have knowledge independent of their current behavior because adults do (as evidenced by their ability to verbalize such knowledge); however, Smith and Thelen (1993) have argued that it is parsimonious to assume that infants have knowledge only as they demonstrate it through their current behavior, because unlike adults, we have no independent measure of what infants know.

\section{Measure}

Reznick and Mandler argue that the $A \bar{B}$ task is an imprecise measure of either memory for locations or object knowledge, because it involves many different processes. The assumption appears to be that infants' knowledge is somehow independent of these processes so that the more of them we peel away, the more we will reveal about infants' knowledge. ${ }^{2}$ In contrast, in the PDP framework, representations are viewed as embedded in processing systems. This neurally motivated view of inseparable knowledge and processing suggests a different tack, namely studying infants' successes and failures given a range of processing demands to gain insight into the nature of their underlying representations. ${ }^{3}$ Indeed, I would argue that such a strategy contributed to Piaget's rich and broad sense of the development of an object concept, rather than a focus on the $A \bar{B}$ task leading him to erroneous conclusions as Mandler argued.

\footnotetext{
${ }^{2}$ To clarify for Russell and Mandler, this is what I meant by 'disembodied knowledge'. I was not, as Russell suspected, arguing that we do away with higher ('personal') level descriptions of developmental phenomena; instead, I was challenging a common assumption about the underlying form of such higher level descriptions.

${ }^{3}$ This tack is clearly not unique to the PDP approach, as evidenced by Baillargeon \& Aguiar's approach in the same spirit.
} 
Russell questions whether the $A \bar{B}$ task taps working memory. Although there is considerable debate about what constitutes working memory (Miyake \& Shah, 1997), one component appears to be the selective representation of task-relevant information. In the $A \bar{B}$ task, information about the toy's most recent hiding location must be selected and maintained in preference to information from previous hidings - not 'an odd kind of working memory' at all.

\section{Reinforcement}

Dehaene argues that reward signals drive learning. In contrast, my model learns based on associations between perceptions, representations, and responses. Although I agree with Dehaene that aspects of the $A \bar{B}$ task may provide infants with reward signals, available data suggest that any such reinforcement does not play a major role in infants' perseverative behavior. First, as noted in the original paper, Diamond (1983) manipulated whether infants were rewarded after making $A \bar{B}$ errors and found 'absolutely no effect of reinforcement on subsequent performance.... When an infant is permitted to play with a toy after an error, he does not perform differently from times when he is allowed no play following an error' (p. 288). Second, as Smith \& Scheier discuss, close analyses of individual performances reveal that all reaches - 'positively reinforced by finding the toy, negatively reinforced by not finding the toy, reaches without an apparent goal' - increase the likelihood of subsequent reaches in the same direction.

\section{Role of modelling}

The commentaries highlight three disagreements about the role of modelling: 1) What can models tell us about cognition and behavior? 2) What assumptions do models make about nature vs. nurture? and 3) What constitutes a fundamental challenge to a model?

\section{Cognition and behavior}

Mandler puts it perhaps most bluntly by saying that my model demonstrates only one way to skin a cat, that is, just because my model perseverates without disembodied knowledge, this does not guarantee that infants do. I agree wholeheartedly with the general point that models cannot determine what underlies behavior. However, PDP models do not merely provide new ways of skinning cats; they can advance the state of our theorizing by demonstrating non-intuitive yet principled ways in which cats may be skinned. First, the non- intuitive: PDP models have demonstrated how double dissociations can arise without modularity (Gonnerman, Andersen, Devlin, Kempler, \& Seidenberg, 1997; Plaut, 1995) and how single dissociations can arise based on the strength of underlying knowledge representations rather than ancillary deficits or separate pathways (Farah, O'Reilly, \& Vecera, 1993; Munakata, McClelland, Johnson, \& Siegler, 1997). Similarly, my $\mathrm{A} \overline{\mathrm{B}}$ model makes a contribution by demonstrating not just another way to think about $A \bar{B}$ errors, but a nonintuitive way that might have otherwise been thought impossible: shared, graded representations and different rates of updating based on these can lead to correct looking to $B$ and perseverative reaching to $A \cdot{ }^{4}$ Second, the principled: the PDP endeavor is not about simply designing systems to mimic behavior. The goal is to explain behavior in terms of a small set of independently motivated computational principles constrained by neurobiology (Seidenberg, 1993). This small set of principles has been applied successfully to understanding behavior across a wide range of domains. The argument is that the resulting models are not arbitrary alternative ways to think about behavior, but wellmotivated ways that are grounded in our understanding of how information processing occurs in the brain.

Of course, even this idealized PDP model has its critics. Mandler reiterates one of McCloskey's (1991) criticisms that it can be difficult to separate the wheat from the chaff in PDP models, that is, to determine which aspects of the model are crucial vs. irrelevant to the proposed theory. In the original paper, I tried to emphasize how the critical aspects of the active-latent theory mapped onto the model. I believe that Mandler is correct in raising questions about other parameters that were not manipulated, but incorrect in seeming to ascribe this problem specifically to the modelling endeavor. As with empirical studies, the modelling endeavor can make progress through the identification of potentially critical variables, the manipulation of these variables, and the measure of effects on the system's behavior. And as with empirical studies, other factors that were not manipulated may contribute to the system's behavior. In both empirical and modelling endeavors, these factors are fleshed out as the field progresses (Smith et al.'s (1995) demonstration of the importance of reaching dynamics in the $A \bar{B}$ task is a good recent empirical example.). However, the exist-

\footnotetext{
${ }^{4}$ Given my lengthy discussion on the nature of the model's representations in the original paper, I confess that I am at a loss as to the basis for Reznick's claim that connectionist models have no representations and so cannot answer fundamental questions about infants' representations.
} 
ence (and eventual discovery) of such additional factors does not negate the earlier demonstration that other critical variables are operative. For example, the importance of reaching dynamics in the $A \bar{B}$ task does not negate the importance of delay; similarly, the nonmanipulated parameters in my model are unlikely to negate the importance of active and latent, graded contributions to the model's $A \bar{B}$ performance.

Finally, Diamond challenges this work as providing less that is new to our understanding of cognition and behavior than I would have readers believe. In the original paper, I explicitly acknowledged that this work has foundations in the work of many other researchers and specified exactly what I viewed as new to this approach to the $A \bar{B}$ task: 1) the treatment of infants' knowledge as embedded in underlying processing mechanisms, 2) the implementation of active and latent traces and their interaction in the form of a PDP network that accounts for infant performance in the $A \bar{B}$ task, and 3) the motivation for the active-latent distinction at the neurophysiological level. Certainly aspects of this approach are shared with existing approaches, but I believe the work makes a novel contribution in unifying these into a single, explicit, model; as far as I can discern, Diamond does not challenge these specified contributions from the work. Moreover, the work is also new in considering the role of memory representations in infants' greater sensitivity to a hidden object's location through gaze-expectation than in reach. Although Diamond's account of the $A \bar{B}$ error has a memory component (as I explained in the original paper and Diamond subsequently elaborated in her commentary), her account focused on inhibition to explain discrepancies between infants' looking and reaching. In contrast, my model demonstrates how inhibitory deficits need not be invoked to explain these discrepancies.

\section{Nature vs. Nurture}

Dehaene argues that my model is endowed with a considerable amount of innate, biologically-determined structure through its prewired connections and the modelling of age through the growth of synaptic strengths. I strongly disagree. First, the prewired connections represent a bias to respond appropriately to location information, which, as I argued in the original paper, infants appear to bring into $A \bar{B}$ studies; they do not learn for the first time how to look where something is happening upon entering such studies. By pointing out that such biases are present by the time infants are tested with the $A \bar{B}$ task, I am in no way claiming that such biases are innately given. Infants have had plenty of opportunity to learn such biases prior to testing. ${ }^{5} \mathrm{Sec}-$ ond, changes in synaptic strengths can occur through maturation or experience; thus, modelling changes in age through this parameter in no way commits me to one or the other factor. My colleagues and I have demonstrated elsewhere how such synaptic changes can arise through experience and lead to stronger representations of hidden objects (Munakata et al., 1997). Increasing synaptic strengths by hand in this model was merely a simplification of this process to demonstrate how such changes can lead to improved performance in the $A \bar{B}$ task. ${ }^{6}$ Finally, Dehaene's claim that my model is too innately endowed (which I dispute) makes the faulty assumption that the 'classical' PDP framework encompasses only nature, not nurture (see Karmiloff-Smith, 1992 for discussion).

\section{Fundamental challenges}

The commentators issued many challenges to the model. I believe that most point to important ways in which the model must be elaborated to account for behavioral data, rather than proposing fundamentally different mechanisms for explaining infant perseveration.

Mareschal and Smith \& Scheier argue for an incorporation of more of the reaching process into the model. As Mareschal argues, the current model falls short of actually specifying the mechanisms that elicit a reach response. For Mareschal, this is an important 'crack in the story' because reaching is discrete (but cf. Diedrich et al., 1997), whereas my model produces only graded responses. For Smith \& Scheier, the reaching process, and bodily movements in general, are critical because they clearly influence subsequent behavior. The influence of prior reaching behaviors is captured in my original model in a simplified manner, and as I argued in the original paper and Smith \& Scheier reiterate, this could be further enriched through the introduction of top-down processing. I agree that a more elaborate representation of the body and the reaching process could help the model to capture more details of infant performance. However, I believe that the lack of such

\footnotetext{
${ }^{5}$ Similarly, when biases of this form are implemented in models of adult cognition (e.g., Cohen, Dunbar, \& McClelland, 1990; Cohen, Romero, Farah, \& Servan-Schreiber, 1994), one would not accuse the authors of claiming that such biases were innately given!

${ }^{6} \mathrm{I}$ argued in the original paper that this gradual increase in synaptic strength across development was more plausible than the sudden 'switching on' of memory units at a single point in development in Dehaene and Changeux's (1989) model. Dehaene's response about sharp onsets of neuronal activity within a task suggests that he thought I was instead criticizing the plausibility of the sudden switching on of units during a task, which I was not.
} 
elaboration in the current model does not detract from the strength of my more general arguments about the contributions of graded, active and latent representations to infant perseveration.

Bremner raises two interesting ways to extend the model. The first is to account for potential individual differences in making long error runs on the $A \bar{B}$ task. I did not present the model with long runs of $B$ trials, nor attempt to simulate individual differences (aside from those across age groups) though others have used PDP simulations to do so (Plaut, in press; MacDonald \& Christiansen, 1997). The second is to extend the model backwards to account for the onset of search (see Munakata et al., 1997) and forwards to account for performance in invisible displacement tasks.

Baillargeon \& Aguiar present data indicating that infants perseverate in a wide variety of paradigms, including visual attention paradigms and object retrieval tasks that do not require infants to remember information about objects. They issue the appropriate challenge that a 'unifying framework for understanding perseveration' account for such phenomena. This challenge strikes me as particularly in the spirit of the modelling endeavor; it points out a limitation in the current model's ability to capture phenomena within the realm of its fundamental claims. Not surprisingly, I agree with Baillargeon \& Aguiar that the active-latent distinction has the potential to provide a mechanism for the range of perseverative phenomena they describe. If the model supports our expectation, our primary disagreement is likely to take the form of whether it is appropriate to describe such a model as a problem solving model.

This is not merely a question of semantics; it is a question of the appropriate level of description for the processes underlying behavior. Several commentators raised the possibility that my model missed an important higher level of description (e.g., Russell's personal level, Mandler's knowledge level, Reznick's psychological level). It is perhaps interesting to note that the issue was not raised by the other modellers (Dehaene and Mareschal) or the dynamic systems theorists (Smith \& Scheier), some of whom argue that such higher level descriptions should be dropped altogether. Although this may seem extreme, it may be equally extreme to wed oneself so strongly to the higher level descriptions that modelling is viewed only as an implementation of them. Progress may depend on a more balanced approach, in which we appreciate the unique contributions of mechanistic and psychological level approaches and use them to productively guide one another.

\section{References}

Ahmed, A., \& Ruffman, T. (1997). Why do infants make A not $B$ errors in a search task, yet show memory for the location of hidden objects in a non-search task?. Manuscript submitted for publication.

Baillargeon, R., DeVos, J., \& Graber, M. (1989). Location memory in 8-month-old infants in a non-search $\mathrm{A} \overline{\mathrm{B}}$ task: Further evidence. Cognitive Development, 4, 345-367.

Baillargeon, R., \& Graber, M. (1988). Evidence of location memory in 8-month-old infants in a non-search $\mathrm{A} \overline{\mathrm{B}}$ task. Developmental Psychology, 24, 502-511.

Bjork, E.L., \& Cummings, E.M. (1984). Infant search errors: Stage of concept development or stage of memory development. Memory and Cognition, 12(1), 1-19.

Bremner, J.G., \& Knowles, L.S. (1984). Piagetian stage IV errors with an object that is directly accessible both visually and manually. Perception, 13, 307-314.

Butterworth, G. (1977). Object disappearance and error in Piaget's stage IV task. Journal of Experimental Child Psychology, 23, 391-401.

Cohen, J.D., Dunbar, K., \& McClelland, J.L. (1990). On the control of automatic processes: A parallel distributed processing model of the stroop effect. Psychological Review, 97(3), 332-361.

Cohen, J.D., Romero, R.D., Farah, M.J., \& Servan-Schreiber, D. (1994). Mechanisms of spatial attention: The relation of macrostructure to microstructure in parietal neglect. Journal of Cognitive Neuroscience, 6(4), 377-387.

Dehaene, S., \& Changeux, J.-P. (1989). A simple model of prefrontal cortex function in delayed-response tasks. Journal of Cognitive Neuroscience, 1(3), 244-261.

Diamond, A. (1983). Behavior changes between 6-12 months of age: What can they tell us about how the mind of the infant is changing? Dissertation Abstracts International, 44(01B), 337. (University Microfilms No. AAD8311882).

Diamond, A., Cruttenden, L., \& Neiderman, D. (1994). A $\bar{B}$ with multiple wells:: 1 . Why are multiple wells sometimes easier than two wells? 2. Memory or memory + inhibition? Developmental Psychology, 30(2), 192-205.

Diedrich, F., Thelen, E., Smith, L., \& Corbetta, D. (1997). Motor memory is a factor in infant perseverative errors. Manuscript submitted for publication.

Farah, M.J., O’Reilly, R.C., \& Vecera, S.P. (1993). Dissociated overt and covert recognition as an emergent property of a lesioned neural network. Psychological Review, 100, 571-588.

Gonnerman, L., Andersen, E., Devlin, J., Kempler, D., \& Seidenberg, M. (1997). Double dissociation of semantic categories in alzheimer's disease. Brain and Language, 57, 254-279.

Harris, P.L. (1974). Perseverative search at a visibly empty place by young infants. Journal of Experimental Child Psychology, 18, 535-42.

Hofstadter, M.C., \& Reznick, J.S. (1996). Response modality affects human infant delayed-response performance. Child Development, 67, 646-658. 
Karmiloff-Smith, A. (1992). Beyond modularity: A developmental perspective on cognitive science. Cambridge, MA: MIT Press.

MacDonald, M., \& Christiansen, M. (1997). Individual differences without working memory: A reply to Just \& Carpenter and Waters \& Caplan. Manuscript submitted for publication.

McCloskey, M. (1991). Networks and theories: The place of connectionism in cognitive science. Psychological Science, 2, 387-395.

Miyake, A., \& Shah, P. (Eds.). (1997). Models of working memory: Mechanisms of active maintenance and executive control. New York: Cambridge University Press. Manuscript in preparation.

Munakata, Y., McClelland, J.L., Johnson, M.H., \& Siegler, R. (1997). Rethinking infant knowledge: Toward an adaptive process account of successes and failures in object permanence tasks. Psychological Review, 104(4), 686-713.

Norman, D., \& Shallice, T. (1986). Attention to action: Willed and automatic control of behavior. In R. Davidson, G. Schwartx, \& D. Shapiro (Eds.), Consciousness and selfregulation: Advances in research and theory, Vol. 4 (pp. 1-18). New York: Plenum.

Plaut, D.C. (1995). Double dissociation without modularity: Evidence from connectionist neuropsychology. Journal of Clinical and Experimental Neuropsychology, 17(2), 291-321.

Plaut, D.C. (in press). Structure and function in the lexical system: Insights from distributed models of word reading and lexical decision. Language and cognitive processes.

Roberts, R., Hager, L., \& Heron, C. (1994). Prefrontal cognitive processes: Working memory and inhibition in the antisaccade task. Journal of Experimental Psychology: General, 123(4), 374-393.

Roberts, R.J., \& Pennington, B.F. (1996). An interactive framework for examining prefrontal cognitive processes. Developmental Neuropsychology, 12(1), 105-126.

Seidenberg, M. (1993). Connectionist models and cognitive theory. Psychological Science, 4(4), 228-235.

Smith, L.B., McLin, D., Titzer, B., \& Thelen, E. (1995). The task dynamics of the A-not-B error. In L.B. Smith (Chair), Tests of a dynamic systems theory: The object concept. Symposium conducted at the 1995 Meeting of the Society for Research in Child Development, Indianapolis, IN.

Smith, L.B., \& Thelen, E. (Eds.). (1993). A dynamic systems approach to development: Applications. Cambridge, MA: MIT Press.

Sophian, C., \& Yengo, L. (1985). Infants' search for visible objects: Implications for the interpretation of early search errors. Journal of Experimental Child Psychology, 40, 260-278.

Wellman, H.M., Cross, D., \& Bartsch, K. (1986). Infant search and object permanence: A meta-analysis of the A-Not-B error. Monographs of the Society for Research in Child Development, 51(3, Serial No. 214).

\section{Author Note}

I thank all of the commentators for their many insights into this work. These provoked a great deal of thought (and writing, some of which was cut due to space constraints - I regret not being able to reply in full to the full range of interesting issues raised). I thank Marshall Haith, Jan Keenan, Randy O'Reilly, David Plaut, Rob Roberts, Jerry Rudy, Tracy Stackhouse and the DU Developmental Lunch Group for useful comments and/or discussion. 